Relations industrielles

Industrial Relations

\title{
Dorsey, Stuart, Christopher Cornwell et David Macpherson, Pensions and Productivity
}

\section{Louis Tremblay}

Volume 54, numéro 2, 1999

URI : https://id.erudit.org/iderudit/051246ar

DOI : https://doi.org/10.7202/051246ar

Aller au sommaire du numéro

Éditeur(s)

Département des relations industrielles de l'Université Laval

ISSN

0034-379X (imprimé)

1703-8138 (numérique)

Découvrir la revue

Citer ce compte rendu

Tremblay, L. (1999). Compte rendu de [Dorsey, Stuart, Christopher Cornwell et David Macpherson, Pensions and Productivity]. Relations industrielles /

Industrial Relations, 54(2), 423-425. https://doi.org/10.7202/051246ar

Tous droits réservés @ C Département des relations industrielles de l'Université Laval, 1999
Ce document est protégé par la loi sur le droit d'auteur. L’utilisation des services d'Érudit (y compris la reproduction) est assujettie à sa politique d'utilisation que vous pouvez consulter en ligne.

https://apropos.erudit.org/fr/usagers/politique-dutilisation/ 
analyse ? L'absence de références devient ainsi une faiblesse importante de cet ouvrage dont on se demande s'il n'est pas essentiellement un assemblage personnel de données éparses et d'histoires, au demeurant, inconnues. L'autre critique que nous formulons est que l'auteur est en proie à des contradictions importantes quant à la démarche plutôt consensuelle qu'il propose pour réussir le passage aux 35 heures. Après avoir maintes fois souligné qu'il s'agit d'un projet collectif, d'entreprise, qui nécessite la mobilisation et l'engagement de tous, l'auteur en vient par ailleurs à formuler aux gestionnaires des suggestions du type : comment contourner les résistances syndicales face à l'ARTT ? De tels propos sont pour le moins étranges eu égard à l'approche de fond suggérée auparavant. Notamment, ces propos ne sont pas tout à fait de nature à faire adhérer l'acteur syndical à ce que l'auteur propose comme un "mode d'emploi" pour réussir le passage aux 35 heures!

\section{JEAN CHAREST \\ École de relations industrielles Université de Montréal}

\section{Pensions and Productivity}

par Stuart DORSEY, Christopher CORNWELl et David MACPHERSON, Kalamazoo, Michigan : W.E. Upjohn Institute for Employment Research, 1998, 139 p., ISBN 0-88099-186-0 (alk. paper) et ISBN 0-88099-185-2 (pbk. : alk. paper).

Les régimes privés de retraite jouent un rôle essentiel pour le remplacement des revenus de travail à la retraite. Généralement, ils sont considérés du point de vue de la demande de régimes de retraite. Les travailleurs demandent des régimes de retraite comme source de revenu à la retraite et les employeurs sont indifférents entre payer un salaire et contribuer à un régime de retraite.

Pour les économistes, les régimes de retraite jouent un rôle plus important dans les lieux de travail. En plus d'être une source de revenu pour la retraite, ils peuvent aussi augmenter la productivité au travail et réduire les coûts de maind'œurve. C'est cette perspective du point de l'offre qui est explorée dans le livre Pension and Productivity de Dorsey, Cornwell et Macpherson. Cette publication couvre des aspects théoriques, institutionnels et empiriques et elle introduit de nouveaux résultats suggérant un lien entre les régimes de retraite et la formation offerte aux employés par l'entreprise.

Ce livre se divise en huit chapitres. Dans l'introduction (chapitre 1), les auteurs cherchent à expliquer les pratiques suivies par les employeurs et les employés quand une relation d'emploi est productive et durable, en adressant des questions comme: Quelle est la base économique sous-jacente aux relations d'emploi durables? Comment les pratiques de rémunération et de promotion contribuent-elles à attirer et à motiver les bons employés ? Quand la relation d'emploi est productive, comment les salaires et les avantages sociaux peuvent-ils simultanément permettre des gains de productivité et décourager les mises à pied et les départs?

Le chapitre 2 constitue un survol de la littérature historique et théorique sur les régimes de retraite. Les auteurs font mention des écrits se rapportant à l'apparition des régimes privés de retraite aux États-Unis et, plus particulièrement, des raisons qui ont amené les employeurs à en offrir (réduction du roulement de la main-d'œuvre, attraction des jeunes travailleurs, imposition d'un âge de retraite obligatoire et, plus récemment, report de l'imposition d'une partie de la rémunération). Ils font état de l'évolution des conditions d'acquisition du droit à une rente en cas de cessation de participation. Ils expliquent que les régimes de retraite, 
particulièrement les régimes à prestations déterminées (RPD) par rapport aux régimes à cotisations déterminées (RCD), constituent des moyens d'assurer une plus grande permanence des employés et de permettre une retraite anticipée.

Le chapitre 3 porte sur les effets recherchés par la mise en place d'un régime de retraite, autant un RPD qu'un RCD. Les RPD ont pour effet d'encourager le service continu et de favoriser la retraite anticipée au moment où la valeur de la rente est à son maximum. Un départ hãtif a pour effet de diminuer sensiblement la valeur des prestations parce que la prestation de départ n'est pas indexée et que les conditions d'acquisition ne sont pas pleinement rencontrées. Le même phénomène se produit pour les participants qui retardent leur retraite, ils ne bénéficient pas de prestations de retraite anticipée qui représentent une partie importante de la valeur de la rente. Au contraire, les RCD sont neutres quant à l'âge de la retraite. Il n'y pas de moment où la valeur du capital est maximale puisque tant que le participant est en emploi, la valeur de son compte croit.

Le chapitre 4 traite des effets des régimes de retraite dans les modèles de marchés internes du travail. L'enjeu fondamental est de voir comment les régimes de retraite peuvent être systématiquement liés à des gains de productivité d'une relation durable d'emploi. Les auteurs observent que l'effet de permanence des régimes de retraite pour les longues relations d'emploi est cohérent quand les habiletés en emploi sont acquises graduellement. Les RPD ont comme qualité de différer une partie de la compensation. Là où les salaires sont inflexibles à la baisse, les RPD peuvent jouer un grand rôle comme indemnités de fin d'emploi lorsque l'employeur améliore les prestations de retraite anticipée.

Les effets des régimes de retraite ne sont pas théoriques. Les pénalités de départ peuvent ètre trop faibles pour les nouveaux engagés, spécialement si la formation est complétée tôt en emploi. D'autres estiment que les pénalités de départ sont trop élevées quand la productivité dans une entreprise diminue. Dans ce cas, des pénalités réduites pourraient inciter les travailleurs à plus de mobilité. Il est admis qu'il est difficile d'établir un mécanisme efficient pour restreindre la mobilité, tout en la favorisant lorsque nécessaire.

Bien que n'ayant pas les mêmes effets que les RPD, les RCD peuvent permettre d'assurer des liens d'emploi avec les travailleurs acceptant qu'une partie de leur rémunération soit différée. Par exemple, les plans $401(\mathrm{k})$, qui ressemblent aux REÉR collectifs, permettent aux employeurs d'augmenter leur contribution au compte des travailleurs qui cotisent les montants maximums permis. Cette façon de faire vise à garder les travailleurs prêts à accepter qu'une partie de leur rémunération soit différée.

Le chapitre 5 porte sur les liens empiriques entre les régimes de retraite et la productivité. Il y a peu de preuves directes que les travailleurs ou les entreprises parties à un régime de retraite soient plus productifs. Cependant, des preuves indirectes montrent une relation forte entre des salaires plus élevés et la couverture d'un régime de retraite. De plus, les travailleurs participant à un régime de retraite quittent moins souvent leur emploi et reçoivent plus de formation.

Ces résultats sont cohérents avec les pénalités importantes imposées lors d'un départ dans les RPD. Cependant, les mêmes résultats sont observés pour les travailleurs et les entreprises participant à un $\mathrm{RCD}$. Ce dernier résultat est aussi cohérent avec l'hypothèse que les RCD attirent les travailleurs prêts à accepter qu'une partie de leur rémunération soit différée. Ce constat pourrait être un des facteurs expliquant l'engouement récent pour les RCD par rapport aux RPD. La possibilité de cibler des travailleurs prêts à accepter qu'une partie de leur rémunération soit différée par une cotisation 
patronale plus élevée, tel que mentionné plus haut, peut être un substitut aux RPD. Malgré cela, les RPD sont plus avantageux eu égard à la productivité et à la retraite anticipée qui peut être offerte aux travailleurs plus âgés.

Le chapitre 6 présente des estimés de la relation entre les régimes de retraite et la formation. Les auteurs ont testé si les régimes de retraite pouvaient augmenter la productivité par des investissements plus importants en formation. Il est clair qu'il y a une relation empirique entre la participation à un régime de retraite et la formation en emploi. Cependant, les données disponibles ne peuvent permettre d'identifier clairement les facteurs explicatifs de cette relation. lls ont trouvé que les travailleurs avec une formation plus poussée sont plus enclins à participer à un RPD.

Le chapitre 7 rapporte les résultats d'un test original de la relation entre la couverture des régimes de retraite et les écarts de productivité. Les auteurs estiment une fonction de production qui inclut comme variable explicative la présence d'un RPD. À partir d'informations concernant un groupe d'entreprises pour les années 1981 à 1992, ils observent une relation importante entre la présence d'un RPD et la productivité du travail. Par ailleurs, au niveau de la productivité totale, les résultats sont plus mitigés. Les écarts moyens de productivité varient de 5 à $8 \%$. Les écarts de productivité tendent à diminuer au cours de la période d'observation. Par exemple, dans certains cas, la différence passe de $15 \%$ en 1981 à $0 \%$ en 1992. Une implication de ce résultat est que l'écart de productivité entre les employeurs ayant un RPD et ceux ayant un RCD tend à diminuer considérablement. Ils signalent que ce résultat peut expliquer la baisse de popularité des RPD au profit des RCD au cours de la même période.
Le chapitre 8 présente un résumé et les conclusions du livre. Les auteurs suggèrent quelques pistes intéressantes de recherche pour le futur. Notamment, ils plaident pour le développement de sources de données plus complètes en vue de mieux connaître les effets des régimes de retraite et des politiques de rémunération sur la productivité. Ils suggèrent aussi que des études devraient être faites sur les effets des RCD. Un autre champ d'étude qui pourrait permettre de mieux connaître les effets des régimes de retraite, mais peu utilisé par les économistes, serait des études de cas. Ils suggèrent que de telles études pourraient être faites chez les firmes qui ont changé leur RPD pour un RCD au cours des dernières années. On pourrait les questionner afin de savoir si un tel changement a eu des effets sur la productivité, les départs, la retraite, et si ces entreprises ont adopté d'autres politiques pour inciter à la retraite anticipée. Ces études de cas pourraient apporter des informations utiles pour aider la recherche quantitative future.

Les auteurs ont réussi à apporter des réponses intéressantes - parfois complètes, d'autres partielles - aux questions qu'ils se sont posées. L'effort et l'originalité de certains tests empiriques rendent cette publication très intéressante. Certains phénomènes qu'ils observent aux ÉtatsUnis, comme la baisse de popularité des RPD au profit des RCD, trouvent écho au Canada et au Québec, avec la baisse de popularité des RPD au profit des REÉR collectifs. Les passages techniques ne devraient pas décourager le lecteur moins familier avec l'arsenal des régimes de retraite et de l'économétrie.

LOUIS TREMBLAY Régie des rentes du Québec 